\title{
ENTROPY INCREASE AS A CONSEQUENCE OF MEASURE INVARIANCE
}

\author{
C. C. BROWN
}

\begin{abstract}
An inequality, used in statistical mechanics for proving that entropy does not decrease, is shown to hold for general $\sigma$-finite measure spaces. We comment briefly on the corresponding Hilbert space result.
\end{abstract}

Let $(\Omega, \mathfrak{A}, \mu)$ be a $\sigma$-finite measure space, $\mathscr{F}_{\mu}$ the family of $\mu$-absolutely continuous measures on $\mathfrak{A}$, and $T: \mathscr{F}_{\mu} \rightarrow \mathscr{F}_{\mu}$ a positive linear and monotone continuous mapping; i.e. $\sup _{i} T\left(\nu_{i}\right)=T\left(\sup _{i} \nu_{i}\right)$ for every monotone sequence, $\nu_{1} \leqslant \nu_{2} \leqslant \cdots$, of elements of $\mathscr{F}_{\mu}$. If $T$ preserves total measure, then every probability measure in $\mathscr{F}_{\mu}$ maps into a probability measure under $T$. If $\rho$ is a probability density with respect to $(\Omega, \mathfrak{A}, \mu)$, then the image of the corresponding probability distribution under $T$ will have a density $\rho^{\prime}$. A question of some interest in statistical mechanics concerns the behavior of integrals of the form $\int \phi(\rho(\omega)) \mu(d \omega)$. Suppose $\phi$ is a convex function defined in the nonnegative reals. Under what conditions is the inequality

$$
\int \phi(\rho(\omega)) \mu(d \omega) \geqslant \int \phi\left(\rho^{\prime}(\omega)\right) \mu(d \omega)
$$

valid? The main case of interest for statistical mechanics is where $\phi(x)=x \log x$ $(x \geqslant 0)$, in which the integral corresponds to the Gibbs entropy [13].

A sufficient condition for the validity of the inequality is essentially the invariance of $\mu$ under $T$. For finite $\mu$ on a countable space $\Omega$, this result can be found in the book of Penrose [12]. The result appears to be attributable to M. J. Klein [10]. A similar result has also been proved for finite $\mu$ on a compact $\Omega$ by J. Voigt [15, Lemma 1.4].

Using an argument that is well known in the theory of probability in connection with the representation of conditional expectations, it is possible to prove the inequality for general $\sigma$-finite spaces. Slightly more generally, let $(\Omega, \mathfrak{A}, \mu)$ and $(\bar{\Omega}, \overline{\mathfrak{A}}, \bar{\mu})$ be $\sigma$-finite measure spaces, and $T$ a monotone continuous positive linear mapping from $\mathscr{F}_{\mu}$ into the set of measures on $\overline{\mathfrak{A}}$. If $T \mu=\bar{\mu}$, then $T \nu$ is $\bar{\mu}$-absolutely continuous for every $\nu \in \mathscr{F}_{\mu}$. If $T \nu(\bar{\Omega})=\nu(\Omega)$ for every $\nu \in \mathscr{F}_{\mu}$, then let $\rho$ be a probability density for $\nu$ and $\rho^{\prime}$ a probability density for $T \nu$.

Received by the editors January 5, 1984 and, in revised form, May 1, 1984.

1980 Mathematics Subject Classification. Primary 28D15: Secondary 82A05, 82A15.

Key words and phrases. Entropy, measure preserving maps. 
THEOREM. For the mapping $T$ as above, let $\rho$ and $\rho^{\prime}$ be the probability densities just defined, and let $\phi$ be a finite-valued convex function defined on $[0, \infty)$. If the integral, $H(\rho):=\int \phi(\rho(\omega)) \mu(d \omega)$, exists and is less than infinity, then the integral, $\bar{H}\left(\rho^{\prime}\right):=\int \phi\left(\rho^{\prime}(\bar{\omega})\right) \bar{\mu}(d \bar{\omega})$, exists and is less than infinity. If both integrals exist in the extended sense, then $H(\rho) \geqslant \bar{H}\left(\rho^{\prime}\right)$.

Proof. Let $\mathscr{L}$ be the set of extended valued nonnegative measurable functions defined with respect to $(\Omega, \mathfrak{A})$, and define $\overline{\mathscr{L}}$ correspondingly for $(\bar{\Omega}, \overline{\mathfrak{A}})$. For $g \in \mathscr{L}$ let $\nu_{g} \in \mathscr{F}_{\mu}$ be the measure on $\mathfrak{A}$ having the density $g$ with respect to $\mu$. Denoting by $\mathscr{L}_{\mu}$ the equivalence classes $\bmod \mu$ of elements of $\mathscr{L}$, the correspondence $g \rightarrow$ $d T\left(\nu_{g}\right) / d \bar{\mu}$ defines a positive linear and monotone continuous mapping $T^{\prime}$ from $\mathscr{L}$ into $\overline{\mathscr{L}}_{\bar{\mu}}$, the set of $\bar{\mu}$-equivalence classes in $\overline{\mathscr{L}} . T^{\prime}$ is also a mapping of the same type from $\mathscr{L}_{\mu}$ into $\overline{\mathscr{L}}_{\bar{\mu}}$. For $E \in \mathfrak{A}$ let $I_{E}$ be the indicator function of $E . T^{\prime}\left(I_{E}\right)$ is represented by a measurable function of $\bar{\omega} \in \bar{\Omega}$, and $I_{\bar{\Omega}} \in T^{\prime}\left(I_{\Omega}\right)$. Using this fact and an argument that is nearly the same as that in Breiman [2, Chapter 4] or Doob [3, Chapter I] for proving the existence of conditional probability kernels, if $f$ is a measurable mapping from $\Omega$ into a $\operatorname{Borel}$ space $(B, \mathcal{B})$, then we can construct a stochastic kernel $K_{f}$ from $(\bar{\Omega}, \overline{\mathfrak{A}})$ to $(B, \mathfrak{B})$ with the property

$$
K_{f}(F, \cdot) \in T^{\prime}\left(I_{F} \circ f\right) \quad(F \in \mathfrak{R}) .
$$

As in the conditional expectation case, one has

$$
\int \phi(y) K_{f}(d y, \cdot) \in T^{\prime}(\phi \circ f)
$$

for every nonnegative extended valued measurable function $\phi$ defined on $(B$, 拸). Consequently,

$$
\rho^{\prime}:=\int|y| K_{\rho}(d y, \cdot)
$$

is a density for $T \nu_{\rho}$. Since $\rho^{\prime}$ is $\bar{\mu}$-almost everywhere finite, we can suppose that $K_{\rho}$ has been so chosen that $\rho^{\prime}$ is everywhere finite. Thus $\int|y| K_{\rho}(d y, \cdot)$ is finite valued and the conditions for an application of Jensen's inequality hold. For the convex function $\phi_{+}(y):=\max [\phi(y), 0](y \in[0, \infty))$, one has

$$
\phi_{+}\left(\rho^{\prime}\right)=\phi_{+}\left(\int y K_{\rho}(d y, \cdot)\right) \leqslant \int \phi_{+}(y) K_{\rho}(d y, \cdot)
$$

and

$$
\begin{aligned}
\int \phi_{+}\left(\rho^{\prime}\right) \bar{\mu}(d \bar{\omega}) & \leqslant \int\left\{\int \phi_{+}(y) K_{\rho}(d y, \bar{\omega})\right\} \bar{\mu}(d \bar{\omega}) \\
& =\int T^{\prime}\left(\phi_{+} \circ \rho\right) \bar{\mu}(d \bar{\omega})=\int \phi_{+}(\rho) \mu(d \omega) .
\end{aligned}
$$

Repeating this argument with $\phi$ in place of $\phi_{+}$, a proof of the theorem as stated is only a matter of technical details.

For a classical mechanical system, let $(\Omega, \mathfrak{A}, \mu)$ be the phase space with the $\mu$-preserving family $\left\{T_{t}\right\}_{t \in R}$ of one-to-one surjective transformations $T_{t}: \Omega \rightarrow \Omega$ 
representing the system flow in $\Omega$. It is often possible to assume that $\mu$ is $\sigma$-finite on the $\sigma$-algebra $\mathscr{T}$ of invariant measurable sets in $\Omega$ (see, for example, [9]). It follows [9] that every initial probability density $\rho_{0}$ in $(\Omega, \mathfrak{A}, \mu)$ has a time average which, in any reasonable definition of approach to equilibrium with time, is equal almost everywhere to the equilibrium density $\rho_{\infty}$ corresponding to $\rho_{0}$. The density $\rho_{\infty}$ is a conditional expectation $E\left(\rho_{0} \mid \mathscr{T}\right)$ of $\rho_{0}$ under $\mu$ with respect to $\mathscr{T}$. Because $\mu$ is $\sigma$-finite on $\mathscr{T}, E(f \mid \mathscr{T})$ is also defined for every nonnegative measurable extended valued function $f$ on $\Omega$. The time average is therefore a restriction of the mapping $T^{\prime}$ from $\mathscr{L} \rightarrow \mathscr{L}_{\mu}$ which derives from the mapping $T: \mathscr{F}_{\mu} \rightarrow \mathscr{F}_{\mu}$ given by $\nu_{g} \rightarrow \nu_{E(g \mid \mathscr{T})}$ ( $g \in \mathscr{L}$ ). This mapping $T$ has the properties demanded by the theorem and is the only such mapping specializing to the average value map.

For quantum mechanical systems, a corresponding result has been proved by $\mathrm{G}$. Lindblad [11]. The following easy consequence of Jensen's inequality seems to simplify Lindblad's proof considerably.

THEOREM. Let $U \subset R$ be an interval, $\phi$ a bounded convex function on $U, A_{1}, A_{2}, \ldots$ nonegative selfadjoint operators in a separable Hilbert space $\mathscr{H}$, with $A_{1}+A_{2}+\cdots$ $=I=$ Identity operator, and $x_{1}, x_{2}, \ldots$ elements of $U$. If $\sum_{\alpha}\left|x_{\alpha}\right| \operatorname{tr} A_{\alpha}<\infty$ then $\phi\left(\sum_{\alpha} x_{\alpha} A_{\alpha}\right)$ is defined as a bounded self adjoint operator in $\mathscr{H}$. If, furthermore, $\operatorname{tr}\left[\sum_{\alpha} \phi\left(x_{\alpha}\right) A_{\alpha}\right]$ exists less than infinity, then

$$
\operatorname{tr}\left[\phi\left(\sum_{\alpha} x_{\alpha} A_{\alpha}\right)\right] \leqslant \operatorname{tr}\left[\sum_{\alpha} \phi\left(x_{\alpha}\right) A_{\alpha}\right] .
$$

\section{REFERENCES}

1. H. Bauer, Wahrscheinlichkeitstheorie und Grudnzüge der Masstheorie, De Gruyter, Berlin, 1978.

2. L. Breiman, Probability, Addison-Wesley, Reading, Mass., 1968.

3. J. L. Doob, Stochastic processes, Wiley, London, 1953.

4. P. und T. Ehrenfest, Zur Theorie der Entropiezunahme in der Statistischen Mechanik von Gibbs, Wien. Ber. 115 (1906).

5. H. Grad, Principles of the kinetic theory of gases, Handbuch der Physik, Springer, Berlin and New York, 1958.

6. K. Jacobs, Lecture notes on ergodic theory, Aarhus Univ., Aarhus, 1963.

7. M. Kac, et al., Probability and related topics in physical sciences, Lectures in Appl. Math. (Proc. Summer Seminar, Boulder, Colo., 1957), Vol. I, Interscience, London and New York, 1959.

8. Foundations of kinetic theory, Proc. Third Berkeley Sympos., Univ. of California Press, Berkeley, Vol. 3, 1954.

9. A. I. Khinchin, Mathematical foundations of statistical mechanics, Dover, New York, 1949.

10. M. J. Klein, Entropy and the Ehrenfest urn model, Physica 22 (1956), 569-575.

11. G. Lindblad, Expectations and entropy inequalities for quantum systems, Comm. Math. Phys. 39 (1974), 111-120.

12. O. Penrose, Foundations of statistical mechanics, Pergamon Press, London, 1970.

13. R. C. Tolman, The principles of statistical mechanics, Oxford Univ. Press, 1938.

14. G. E. Uhlenbeck and G. W. Ford, Lectures in statistical mechanics, Lectures in Appl. Math., Vol. 1, Amer. Math. Soc., Providence, R. I., 1963.

15. J. I. Voigt, Stochastic operators, information and entropy, Comm. Math. Phys. 81 (1981), 31-38.

16. A. Wehrl, General properties of entropy, Rev. Modern Phys. 50 (1978), 221-260.

Mathematics Institut, Freie Universität Berlin, Arnimallee 2 - 6, Berlin 33, West Germany 